\title{
Quantitative assessment of gastric atrophy using the syntactic structure analysis
}

\author{
A M Zaitoun, $\mathrm{H}$ Al Mardini, C O Record
}

\begin{abstract}
Aim-To assess the topographical relation between gastric glands, using the minimum spanning tree (MST), to derive both a model of neighbourhood and quantitative representation of the tissue's architecture, to assess the characteristic features of gastric atrophy, and to assess the grades of gastric atrophy.

Methods-Haematoxylin and eosin stained sections from corporal and antral biopsy specimens $(n=139)$ from normal patients and from patients with nonatrophic gastritis and atrophic gastritis of grades 1, 2, and 3 (Sydney system) were assessed by image analysis system (Prodit 5.2) and 11 syntactic structure features were derived. These included both line and connectivity features.
\end{abstract}

Results-Syntactic structure analysis was correlated with the semiquantitative grading system of gastric atrophy. The study showed significant reductions in the number of points and the length of MST in both body and antrum. The standard deviation of the length of MST was significantly increased in all grades of atrophy. The connectivity to two glands was the highest and most affected by the increased grade of atrophy. The reciprocal values of the Wiener, Randic, and Balaban indices showed significant changes in the volume of gland, abnormality in the shape of glands, and changes in irregularity and branching of the glands in both types of gastric mucosa. There was a complete separation in the MST, connectivity, and index values between low grade and high grade gastric atrophy

Conclusions-(1) Gastric atrophy was characterised by loss of the gland, variation in the volume, reduction in the neighbourhood, irregularity in spacing, and abnormality in the shape of the glands. (2) Syntactic structure analysis significantly differentiated minor changes in gastric gland (low grade atrophy) from high grade atrophy of clinical significance. (3) Syntactic structure analysis is a simple, fast, and highly reproducible technique and appears a promising method for quantitative assessment of atrophy.

(F Clin Pathol 1998;51:895-900)

Keywords: gastritis; atrophy; Sydney system; tissue architecture; minimum spanning tree

Gastric mucosa is made up of structural and functional glands. The normal mucosa of the body of the stomach is made up of tightly packed glands with short foveolae. The normal antral mucosa consists of deep foveolae with larger glands than those of the body mucosa. The glands in both body and antrum are closely adjacent with each other and use space in an optimal way with respect to their specific function and the surrounding lamina propria. The topographies of gastric glands in either the body or the antrum must be considered to be linked to morphogenesis, structural stability, and functional status.

Gastric atrophy is defined by loss of glandular tissue. However, the presence of oedema, inflammatory cellular infiltrate, and lymphoid follicles in the lamina propria may lead to displacement of the glands, making the evaluation of gastric atrophy rather difficult in certain cases. In the Sydney system of classification of gastritis, atrophy was graded semiquantitatively into three grades. ${ }^{12}$ Although other graded variables of this system can be evaluated with a reasonable degree of accuracy, ${ }^{3}$ the interobserver degree of agreement for atrophy may not be satisfactory. There is therefore a need for a quantitative method to reduce interobserver variation.

Syntactic structure analysis is a technique based on the graph theory. It is used to analyse morphometric features in a two dimensional space by measuring properties of the structures, and by analysis of the geometrical relations between the structures. ${ }^{4}$ It is used to obtain quantitative information about tissue architecture. This technique has many applications in histology and cytology. ${ }^{6-19}$ In histopathology, syntactic structure analysis has been used to differentiate mesothelioma from metastatic carcinoma of the pleura ${ }^{10}$ and provides clinically relevant information in carcinoma of the lung, ${ }^{14-16}$ prostate carcinoma, ${ }^{15}$ colonic adenoma and carcinoma, ${ }^{12}$ colonic dysplasia, ${ }^{16}$ carcinoma of the breast, ${ }^{9}$ endometrial carcinoma, ${ }^{17}$ and soft tissue neoplasms. ${ }^{18}$ To the best of our knowledge, no study using syntactic structure analysis has been described previously assessing gastric atrophy.

Our approach is an attempt to study the glandular population at the "sociological" level - that is, at the level of the spatial organisation in interrelationship with the gastric mucosa in a normal healthy stomach, and in chronic inflammation with and without atrophy. We sought also to assess the characteristic features of gastric atrophy and to grade atrophy into two grades.

Methods

Gastric biopsy specimens from patients $(n=126)$ with ulcer and non-ulcer dyspepsia were used. The pathological changes were
Accepted for publication 21 July 1998 
Table 1 Number of cases used in five diagnostic categories in relation to topography from 126 patients with peptic dyspepsia. Biopsy specimens from 13 patients showed atrophy in both corpus and antral mucosa were assessed separately

\begin{tabular}{lll}
\hline Diagnostic categories & Corpus & Antrum \\
\hline Normal & 15 & 9 \\
Non-atrophic gastritis & 15 & 20 \\
Grade I atrophic gastritis & 11 & 20 \\
Grade II atrophic gastritis & 10 & 15 \\
Grade III atrophic gastritis & 9 & 15
\end{tabular}

divided into five diagnostic categories (table 1): normal, chronic gastritis without atrophy, and chronic gastritis with grade 1 , grade 2 , and grade 3 atrophy (Sydney system for classification of chronic gastritis ${ }^{12}$ ).

Specimens were fixed with $4 \%$ formol saline. Haematoxylin and eosin sections were analysed ( $5 \mu \mathrm{m}$ thickness). The theoretical basis of syntactic structure analysis has been extensively discussed before. ${ }^{671319-22}$ Syntactic structure analysis was performed in at least 10 fields using a $\times 4$ objective. Under this magnification, the microscopic field contained the entire thickness of the mucosa. To avoid the boundary effect, the area of measurement did not include the superficial epithelium of gastric mucosa and areas with lymphoid follicles and

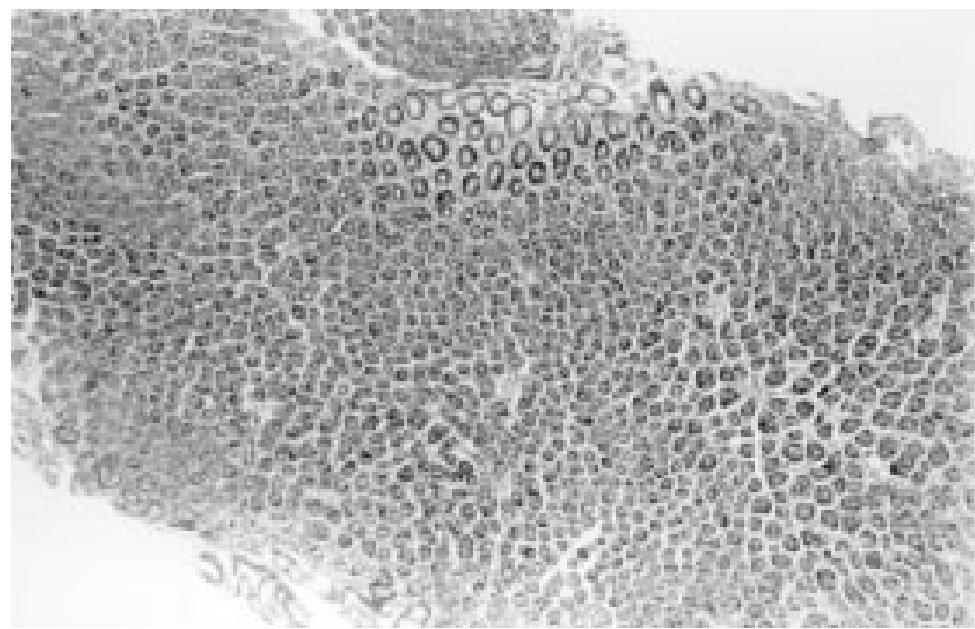

Figure 1 Normal body type gastric mucosa. Small uniform glands with minimal lamina propria are seen. Haematoxylin and eosin stain, $\times 31$.



Figure 2 Normal antral type gastric mucosa. The glands are uniform in size and the distance between glands is regular. Haematoxylin and eosin stain, $\times 31$. occasional areas with straight long glands were also excluded.

Sections were cut at a perpendicular and tangential angle and included the superficial epithelium and muscularis mucosae. Each slide contained three to five sections (9-15 sections were examined). The centre of gravity of all glands was interactively marked on a video screen using a cursor. Using this set of points, the computer composed the minimum spanning tree (MST). The following syntactic structure features were derived from the MST compound in each field: number of points; length of the MST; average length; minimum length; maximum length; and number of points with one, two, three, four, and five neighbours. The average length is obtained by dividing the total length by the number of points. For each of these, the mean, standard error, and standard deviation were calculated, together with other MST features: the Wiener index, the Randic connectivity index, and the Balaban centric index. The changes in volume of the gastric glands were assessed by the Weiner index, the shape of the glands was assessed by the Randic index, and the degree of branching and irregularity of the glands was assessed by the Balaban index. The reciprocal values of these indices were used since the study describes a declining population of glands, unlike tumours where the population of cells or glands is increasing in exponential phase.

Syntactic structure analysis was performed using the PRODIT digitising interactive video overlay system (BMA, The Netherlands) which is composed of a CD camera mounted on a Nikon microscope (Labophot 2), connected to

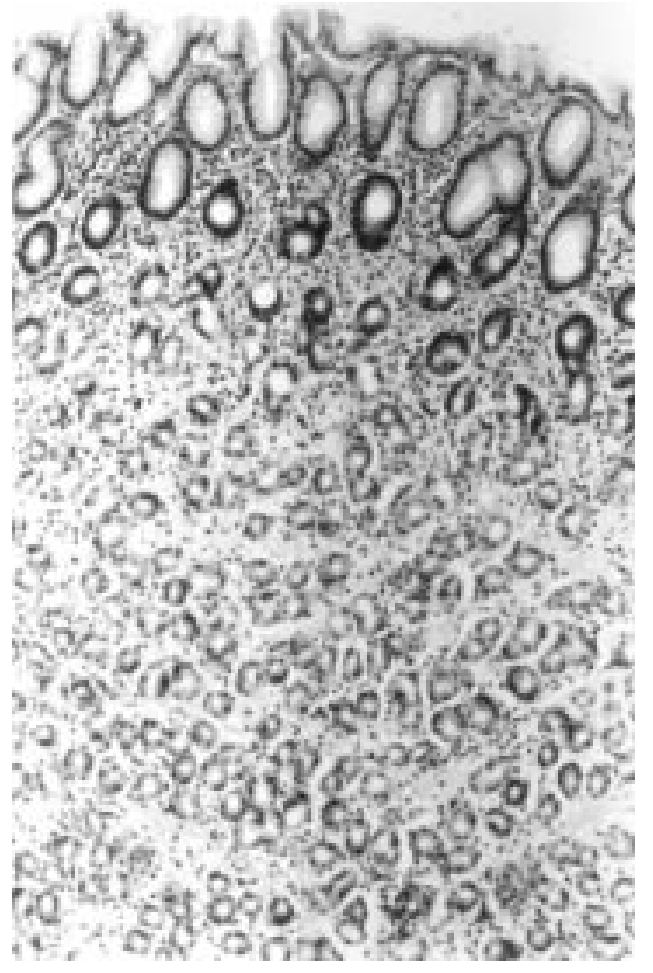

Figure 3 Non-atrophic corporal gastritis. The architecture of the crypts is normal. There is an increase in the area of lamina propria. Haematoxylin and eosin stain, $\times 77$. 




Figure 4 Antral type gastric mucosa with grade III atrophy showing marked loss and irregularity in spacing of the glands. Haematoxylin and eosin stain, $\times 77$.

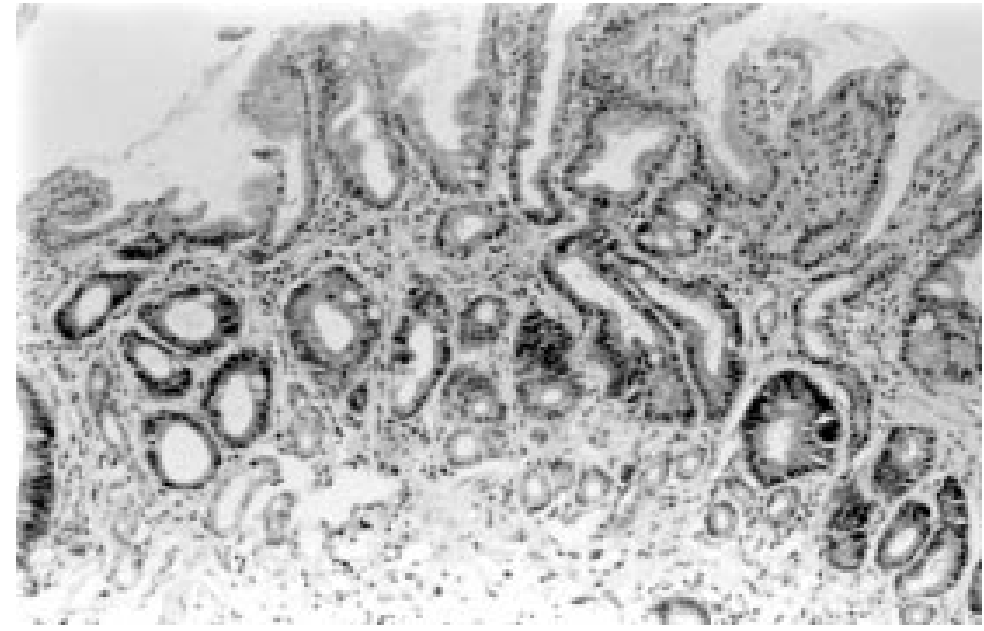

Figure 5 Gastric mucosa with grade II atrophy showing variation in the size of the glands. Haematoxylin and eosin stain, $\times 77$.

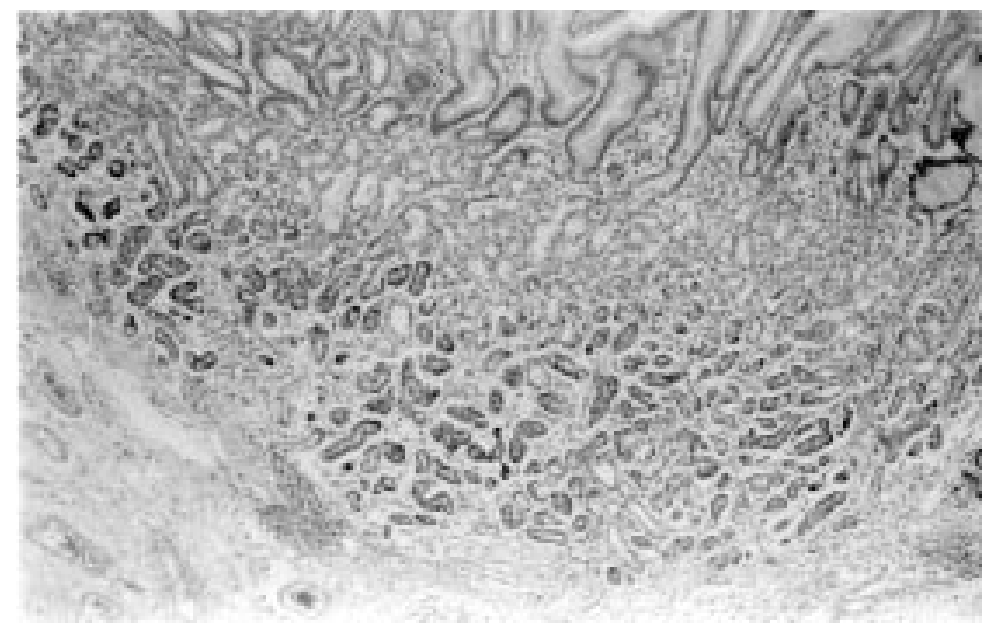

Figure 6 Antral mucosa with grade I atrophy showing abnormality in the shape of the glands. Haematoxylin and eosin stain, $\times 31$.

a video screen in a personal computer and special software.

STATISTICAL ANALYSIS

The mean and standard deviation values were obtained using a software program (SPSS for Windows 6.1). Analysis of variance (ANOVA) was used to derive $\mathrm{p}$ values (assuming normally distributed data). The level of significance was set to $\mathrm{p}<0.05$. The intraobserver reproducibility was tested in two ways. First, the intrafield variation was tested by measuring five fields from one patient and each was measured five times. Second, five patients were measured twice. The reproducibility was calculated for MST. This was $97 \%$ for the intrafield variation and $95.6 \%$ for the intraobserver variation. The values of MST features, connectivity indices, and reciprocal of Wiener, Balaban, and Randic indices for grade II gastric atrophy (Sydney system) were divided into two groups in relation to the median values. Values below the median were computed to the corresponding values of grade I atrophy (low grade atrophy) and all the values above the median were regrouped with grade III atrophy (high grade atrophy). The statistical significance between these two grades was also obtained.

\section{Results}

MORPHOLOGICAL FEATURES

Figures 1 and 2 show histological sections from normal body and antrum respectively. Figure 3 shows chronic gastritis without atrophy. The main morphological features of gastric atrophy are shown in figs $4-7$. These include loss of gland, irregularity in spacing with loss of connectivity (fig 4), variation in the volume (fig 5), abnormality in the shape (fig 6), and branching of the glands (fig 7 ).

QUANTITATIVE FEATURES

Figure 8 shows a screen picture from normal body type mucosa (A), and chronic gastritis with grade I (B), grade II (C), and grade III (D) atrophy. The total of length of MST was significantly reduced $(p<0.001)$ in both body and in antral mucosa from grade I to grade III atrophy (fig 9). Figure 10A shows the number of points, in both body and antral mucosa, in the three grades system. The number of points in body-type mucosa was reduced from 168 in normal to 108 in inflamed mucosa without atrophy and to 30 with grade III atrophy. Similar changes were seen in antral mucosa. The differences between groups was highly significant $(p<0.001)$. Figure 10B shows the standard deviation of the MST in both body and antral-type mucosa. In normal and inflamed mucosa the points are situated very close to each other. In gastric atrophy there is a wide scatter of the points leading to increase in SD $(\mathrm{p}<0.001)$. The average of the MST in both types of mucosa is shown in fig 10C. There was a significant increase in the average values of MST from normal mucosa to grade III gastric atrophy $(p<0.001)$. The number of neighbours connected to each gland is shown in fig 11. In antral mucosa the connectivity to two glands was the highest. The connectivity for two neighbours was reduced from 90 in normal mucosa to 40 in grade I and to 30 in grade III atrophy. (The $\mathrm{p}$ value for connectivity to two glands was $<0.001$ for body and antral mucosa.)

The reciprocal values of Wiener, Randic, and Balaban indices for body and antral mucosa are 



Figure 8 A screen picture from normal body mucosa $(A)$, and chronic gastritis with grade I (B), grade II (C), and grade III atrophy (D), illustrating the features of the minimum spanning tree. The total distance between the glands represents the total length of the tree. $\times 4$ objective was used
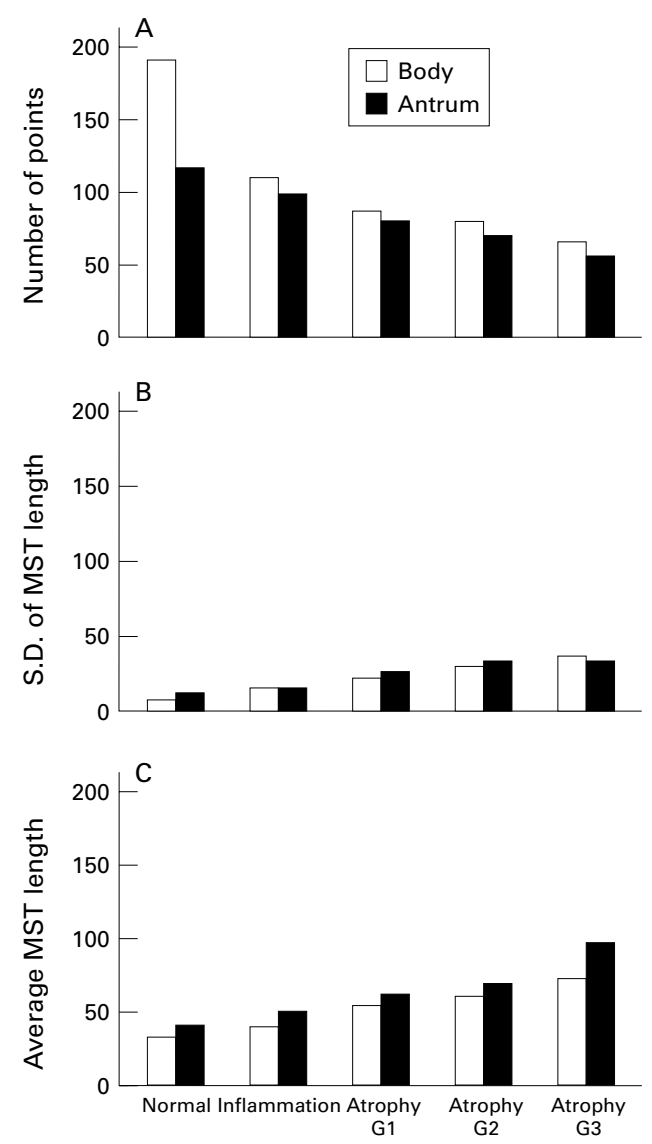

Figure 10 (A) The mean number of points for body mucosa and antral mucosa in normal, chronic inflammation without atrophy, and with grades I, II, and III atrophy. (B) The standard deviation of the minimum spanning tree in body type and antral type mucosa in normal, chronic inflammation without atrophy, and with grades I, II, and III atrophy. (C) The average length of MST for body and antral gastric mucosa in normal,

chronic inflammation without atrophy, and with grades I, II, and III atrophy.

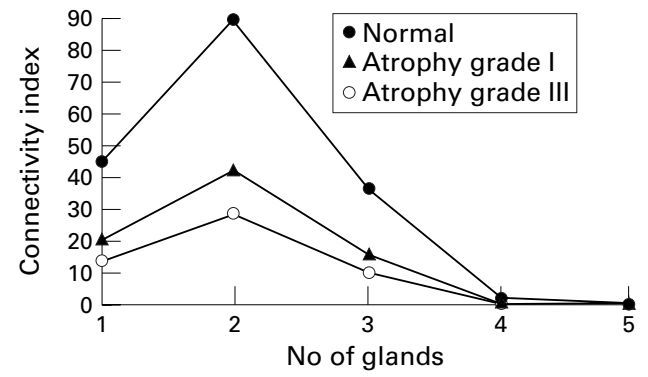

Figure 11 The connectivity to one gland (C1), two glands (C2), three glands (C3), four glands (C4), and five glands (C5) in normal mucosa and grade I and III gastric atrophy of the antrum.

to grade III atrophy $(\mathrm{p}<0.001)$. The Balaban index showed significant changes in branching and irregularity of the glands with the increased grade of atrophy in both body and antrum $(\mathrm{p}<0.001)$.

Figure 9 The total length of the minimum spanning tree in body mucosa and antral mucosa in normal, chronic inflammation without atrophy and with grades I, II, and III atrophy.

shown in fig $12 \mathrm{~A}, \mathrm{~B}$, and $\mathrm{C}$, respectively). There was a significant change in the volume of the glands from normal to grade III atrophy in both body and antral mucosa $(\mathrm{p}<0.001)$. The Randic connectivity index showed significant changes in the shape of the glands from normal
TWO GRADE SYSTEM OF GASTRIC ATROPHY

The results of reclassifying quantitative features of gastric atrophy into a two grade system, low grade and high grade, are shown in table 2 . There was a twofold decrease from low to high grade in the number of points in the 

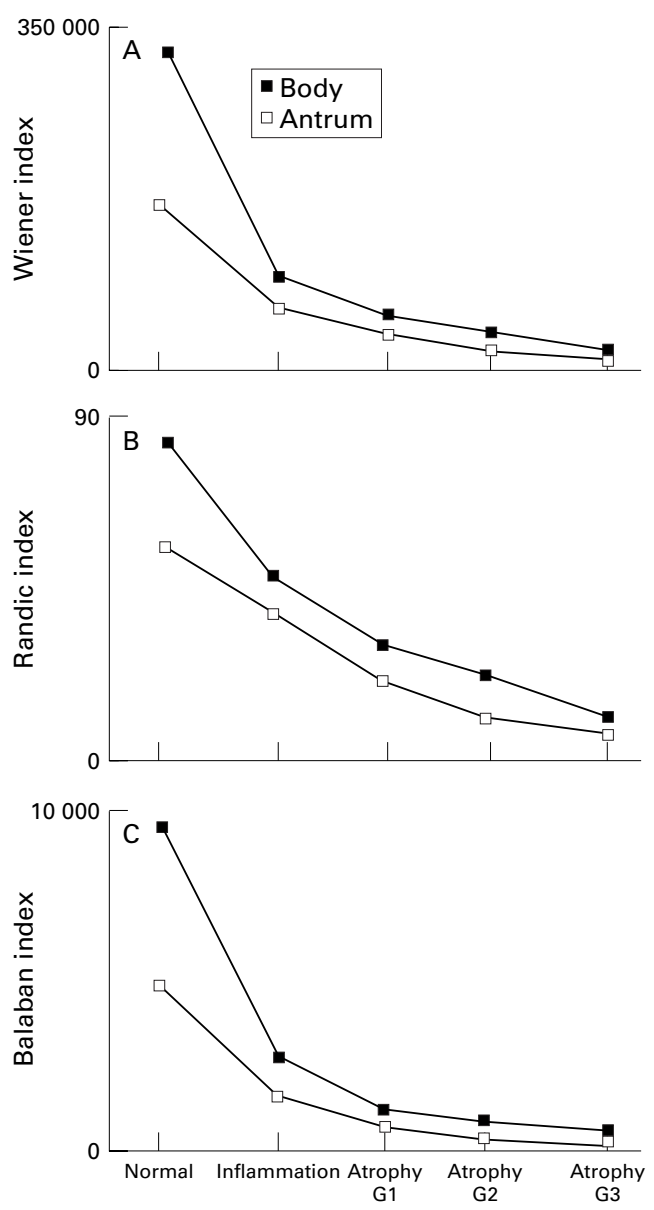

Figure 12 (A) The mean reciprocal values of $(A)$ the Wiener index, $(B)$ the Randic index, and $(C)$ the Balaban index in both body and antrum in normal stomach, chronic inflammation without atrophy, and grades I, II, and III atrophy.

body mucosa $(\mathrm{p}<0.001)$, and a 1.5 -fold decrease in this value in the antrum $(\mathrm{p}<0.001)$. The connectivity to two glands was significantly reduced by 1.5 -fold from low grade to high grade atrophy in both body and antrum $(\mathrm{p}<0.001)$.

Table 2 Minimum spanning tree values with connectivity indices for body-type and antral-type mucosa, using a two grade system for gastric atrophy

\begin{tabular}{|c|c|c|c|c|c|}
\hline & \multicolumn{2}{|c|}{ Low grade atrophy } & \multicolumn{2}{|c|}{ High grade atrophy } & \multirow[b]{2}{*}{ p Value } \\
\hline & Mean & $S D$ & Mean & $S D$ & \\
\hline \multicolumn{6}{|c|}{ Number of points } \\
\hline Body & 79 & 22 & 41 & 18 & * \\
\hline Antrum & 69 & 8 & 46 & 10 &  \\
\hline \multicolumn{6}{|l|}{ MST length } \\
\hline Body & 4123 & 508 & 3144 & 530 & $\star$ \\
\hline Antrum & 3642 & 235 & 2878 & 378 & $\star \star \star \star$ \\
\hline \multicolumn{6}{|c|}{ Standard deviation } \\
\hline Body & 18 & 3 & 26 & 5 & $\star$ \\
\hline Antrum & 20 & 2 & 26 & 5 & $\star \star \star \star$ \\
\hline \multicolumn{6}{|c|}{ Connectivity to two glands } \\
\hline Body & 46 & 10 & 28 & 11 & * \\
\hline Antrum & 36 & 5 & 24 & 5 & $\star \star \star \star$ \\
\hline \multicolumn{6}{|c|}{ Wiener index } \\
\hline Body & 60446 & 42261 & 20281 & 22059 & * \\
\hline Antrum & 30618 & 10875 & 11549 & 5774 & 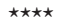 \\
\hline \multicolumn{6}{|c|}{ Balaban index } \\
\hline Body & 2647 & 1665 & 1005 & 762 & * \\
\hline Antrum & 1390 & 327 & 697 & 321 & $\star \star \star$ \\
\hline \multicolumn{6}{|c|}{ Randic index } \\
\hline Body & 41 & 9 & 26 & 9 & $\star \star$ \\
\hline Antrum & 32 & 4 & 21 & 5 & 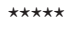 \\
\hline
\end{tabular}

The reciprocal values of all indices (Wiener, Balaban, and Randic) were significantly reduced by 1.5 - to threefold from low grade to high grade atrophy $(\mathrm{p}<0.001$ in all cases) indicating significant changes in the volume, shape, irregularity, and branching of the glands in gastric atrophy.

\section{Discussion}

Gastric atrophy is defined by loss of glands resulting from inflammatory erosion and ulceration of the gastric mucosa. It is most commonly seen in long standing Helicobacter pylori associated gastritis and idiopathic gastritis. Gastric atrophy in both antrum and body of the stomach may be classified into three grades. ${ }^{1}$ In this study we have used a quantitative approach to assess gastric atrophy. Most quantitative techniques operate on high resolution levels and provide information on cellular, nuclear, and nucleolar features. Stereology and syntactic structure analysis are low resolution techniques that can yield quantitative data on tissue architecture. In this technique the cutting angle has particular significance. Although the regularity of the position of glands in normal gastric mucosa is independent of the cutting angle, in this stereological syntactic technique, whether the angle is perpendicular or tangential has a marked bearing on the quantitative parameters for the specimens. To the best of our knowledge, this is the first study analysing the geometrical connection between gastric glands at low magnification. The advantage of using a low magnification ( $\times 4$ objective) is that it allows us to scan a large area of the specimen. Using a similar procedure at low magnification, Kayser et al obtained significant results for differentiating adenoma from carcinoma of the colon. ${ }^{12}$ This is the first study to describe the changes in the population of glands in decline. Most other studies ${ }^{10-18} 21$ have employed the technique to study changes in exponential growth pattern in premalignant conditions and cancer.

Our study has shown significant differences in several features of syntactic structure and total length of the minimum spanning tree, which indicate loss of gastric glands that characterise gastric atrophy. In normal and inflamed gastric mucosa the crypts are closely related to each other with respect to their geometrical properties. However, in chronic gastritis with atrophy, there is loss of glands with wide scatter of the remaining glands, indicating variation in the distance between the glands and in the length of the minimum spanning tree. The study showed that the connectivity between gastric glands is reduced by $50 \%$ in grade I and $60 \%$ in grade III gastric atrophy. The loss of connectivity could be explained by the inflammatory changes in the lamina propria but was caused by the actual loss of epithelial tissue within the mucosa.

As well as demonstrating changes in the number of glands and their geometric relationship, our study has shown that there was no variation in the size of the glands in normal mucosa, minimal variation in inflamed mucosa, and marked variation in gastric atrophy. 
This would indicate that the process of atrophy varies between glands and groups of glands. Another feature of the mucosa in gastric atrophy was the change in the shape of the gland, which became more marked with the increasing grade of atrophy. When regeneration occurs after loss of glands and inflammation, some of the crypts become irregular and "branched", as the change in the Balaban index has shown.

In the original Sydney system of classification of chronic gastritis, atrophy was graded into three grades. ${ }^{12}$ In the updated Sydney system, gastritis was classified as atrophic and non-atrophic. ${ }^{23}$ The degree of agreement among gastric pathologists in the grading of morphological features of $H$ pylori gastritis is reasonable. However, the interobserver agreement for grading atrophy is often poor. ${ }^{3}$ We have therefore attempted to divide gastric atrophy into two grades, low grade and higher grade. The study has shown almost complete separation in the quantitative values of atrophy between these two grades. Although recognising low grade gastric atrophy is difficult by morphological assessment, ${ }^{23}$ quantitative analysis can easily show the minor features of atrophy. It is generally believed that high grade gastric atrophy associated with intestinal metaplasia and dysplasia has prognostic clinical significance. ${ }^{24}{ }^{25} \mathrm{~A}$ minor degree of gastric atrophy may not have any clinical significance, and its natural history is difficult to evaluate. It is therefore important to divide gastritis into non-atrophic and atrophic types and to grade gastric atrophy with a reasonable degree of accuracy. In addition to distinguishing minor degrees of atrophy from high grade atrophy, quantitative methods would improve the documentation of gastric atrophy and serve to reduce interobserver variation.

We conclude that syntactic structure analysis is an objective quantitative technique for assessing gastric atrophy. Atrophy of both corporal and antral gastric mucosa was characterised by loss of glands, irregularity of spacing, and abnormality of the shape of the glands. Regrading gastric atrophy into low grade and high grade may help in differentiating minor degrees of atrophy (which have no clinical significance) from high grade atrophy which does have clinical significance.

1 Price $\mathrm{AB}$. The Sydney system: histological division. $f$ Gastroenterol Hepatol 1991;6:209-22.
2 Zaitoun AM. Histological study of chronic gastritis from the United Arab Emirates using the Sydney system of classifiUnited Arab Emirates using the Sydney
cation. $\mathcal{F}$ Clin Pathol 1994;47:810-15.

3 Andrew A, Wyatt JI, Dixon MF. Observer variation in the assessment of chronic gastritis according to the Sydney System. Histopathology 1994;25:317-33.

4 Marcelpoil R, Usson Y. Methods for the study of cellular sociology: Voronoi diagrams and parameterisation of the spatial relationship. F Theor Biol 1992;154:359-69.

5 Kayser K. Neighbourhood condition and application of syntactic structure analysis in histopathology. Acta Stereol 1987;6:373-84

6 Van Diest PJ, Fleege JC, Baak JPA. An introduction to syntactic structure analysis. Il Friuli Medico Alpe Adria fournal of Medicine 1992;47:221-8.

7 Van Diest PJ, Kayser K, Meijer GA, et al. Syntactic structure analysis. Pathologica 1995;87:255-62.

8 Gilles F, Gentile A, Le Doussal V, et al. Grading of cystosacoma phyllodes by texture analysis of tissue architecture. Anal Quant Cytol Histol 1994;16:95-100.

9 Van Diest PJ, Fleege JC, Baak JPA. Syntactic structure analysis in invasive breast cancer: Analysis of reproducibility, biologic background and prognostic value. Hum Pathol 1992;23:876-83.

10 Kayser K, Bosslet K, Ebert W, et al. Differential diagnosis of mesothelioma and metastatic adenocarcinoma of pleura by means of indirect immunoperoxidase technique combined with syntactic structure analysis. Tumour Diagn Ther 1987; 8:28-35.

11 Kayser K, Stute H, Bubenzer J, et al. Combined morphometrical and syntactic structure analysis as tools for histomorphological insight into human lung carcinoma growth. Anal Cell Pathol 1990;2:167-78.

12 Kayser K, Shaver M, Modlinger F, et al. Neighborhood analysis of low magnification structures (glands) in healthy, adenomatous and carcinomatous colon mucosa. Pathol Res Pract 1986;181:153-8.

13 Kayser K, Kiefer B, Toomes H, et al. Analysis of adenomatous structures in histopathology. Anal Quant Cytol Histol 1987;9:273-8.

14 Kayser K, Liewald F, Kremer K, et al. Alteration of integrated optical density and intercellular structure after induction chemotherapy and survival in lung carcinoma patients treated surgically. Anal Quant Cytol Histol 1994;16: $18-24$

15 Kayser K, Fitzer M, Bulzebruck H, et al. TNM stage, mmunohistology, syntactic structure analysis and survival in patients with small cell anaplastic carcinoma of the lung. 7 Cancer Res Clin Oncol 1987;113:473-80.

16 Bibbo M, Kim DH, di Loreto C, et al. Tissue architecture features for the grading of prostatic carcinoma. Anal Quant Cytol Histol 1990;12:229-36.

17 Meijer GA, Van Diest PJ, Fleege JC, et al. Syntactic structure analysis of the arrangement of nuclei in dysplastic structure analysis of the arrangement of nuclei in dysplastic Cytol Histol 1992;14:491-8.

18 Tezuka F, Sato I, Higashiiwai H. Method for the quantitative evaluation of the distribution pattern of nuclei in normal and malignant endometrial epithelium. Anal Quant Cytol Histol 1990;12:237-41.

19 Kayser K, Sandau K, Bohm G, et al. Analysis of soft tissue tumours by an attributed minimum spanning tree. Anal Quant Cytol Histol 1992;13:329-34.

20 Kayser K, Hoffgen H. Pattern recognition in histopathology by order of textures. Med Inform 1984;9:55-9.

21 O'Callaghan JF. An alternative definition for "Neighborhood of a Point". IEEE Trans Comput 1975;24:1121-5.

22 Marcelpoil R. Normalization of the minimum spanning tree. Anal Cell Pathol 1993;5:177-86.

23 Dixon MF, Genta RM, Yardley JH, et al, and the participants in the International Workshop on the Histopathology of Gastritis, Houston 1994. Classification and grading of gastritis. The updated Sydney system. Am F Surg Pathol 1996;20:1161-81.

24 Rokkas T, Filipe MI, Sladen GE. Detection of an increased incidence of early gastric cancer in patients with intestinal me are closely followed up. Gut 1991; 32:1110-13.

25 Siurala M, Lehtola J, Sipponen P. Atrophic gastritis and its sequelae. Scand $\mathcal{F}$ Gastroenterol 1974;9:441-6. 